\title{
Mutual Interaction of Red Blood Cells Assessed by Optical Tweezers and SEM Imaging
}

\author{
Tatiana Avsievich, ${ }^{*}$ Alexey Popov,* Alexander Bykov, and Igor Meglinski
}

Opto-Electronic and Measurement Techniques, University of Oulu, P.O. Box 4500, Oulu, 90014, Finland

*Corresponding author: tatiana.avsievich@oulu.fi and/or alexey.popov@oulu.fi

Received XX Month XXXX; revised XX Month, XXXX; accepted XX Month XXXX; posted XX Month XXXX (Doc. ID XXXXX); published XX Month XXXX

\begin{abstract}
The adhesion of RBC has been studied extensively in frame of cell-tocell interaction induced by dextran macromolecules, whereas the data is lacking for native plasma solution. We apply optical tweezers to investigate the induced adhesion of RBC in plasma and in dextran solution. Two hypotheses, cross-bridges and depletion layer are typically used to describe the mechanism of cells interaction, while both need to be confirmed experimentally. These interactions in fact are very much dependent on size and concentration of dextran and proteins in plasma. The results show that in different dextran solutions the interaction of adhering RBC agrees well with the quantitative predictions obtained based on the depletion-induced cells adhesion model, whereas a migrating cross-bridges model is more appropriate for plasma. Despite the different mechanisms of RBC interaction in a mixture of dextran with the size ranges and volume fraction proportional to plasma proteins the dependence of RBC adhering tends to be close to the cross-bridges model. The induced aggregation of RBC in the dextran solutions and in native plasma are observed by direct visualization utilizing scanning electron microscopy (SEM). (C) 2018 Optical Society of America
\end{abstract}

OCIS codes: (170.1470) Blood or tissue constituent monitoring; (170.1530) Cell analysis; (170.2655) Functional monitoring and imaging; (350.4855) Optical tweezers or optical manipulation.

http://dx.doi.org/

Mutual interactions of red blood cells (RBCs), including their reversible aggregation, strongly influence rheological properties of blood. The spontaneous aggregation of RBC is a high risk factor known in a number of pathologies, including malaria, diabetes mellitus, hypertension, inflammation and others [1]. For decades, fundamental studies of RBC aggregation have been attracting a significant attention from various viewpoints ranging from basic biophysical examination to perspectives of clinical application. However, the basic mechanisms of this process are still not thoroughly understood.

Various techniques have been utilized for quantitative characterization of RBC aggregation. The dynamic light scattering was applied for assessment of aggregation of an ensemble of RBCs for diagnostic purposes [2]. Conventional microscopy, since its first application, is widely used to study the relation of RBC aggregation to pathological conditions [3,4]. Micropipette aspiration technique (MAT) was successfully employed for measuring energy of cell-tocell interaction [5]. Scanning electron microscopy (SEM) is routinely utilized for direct observation of cells interaction, their deformation and variations of intercellular distances [6]. Atomic force microscopy (AFM) is used effectively for quantitative assessment of the parameters of RBC adhesion [7].

Introduced by Ashkin, the trapping of micro-particles with a sharply focused laser beam [8], so-called optical tweezers (OT), is now becoming a popular tool, in particular for studying the interaction of cells $[9,10]$. OT allows to measure the forces of interaction between cells with up to pico-Newton $(\mathrm{pN})$ resolution, providing an opportunity for a precise control of the cells contact [11]. This approach provides a high potential to gain new insights in the mechanisms of RBC interaction. The OT technique developed in-house [12,13] were used in the current study to examine mutual interaction of RBC and its dependence on size and concentration of dextran and proteins in plasma.

In blood plasma the extracellular factors affecting aggregation of $\mathrm{RBC}$ are represented by various blood proteins, among which are fibrinogen, globulins, C-reactive proteins [1]. Neutral dextran molecules suspended in a phosphate buffered saline (PBS), are used to artificially trigger the aggregation of $\mathrm{RBC}$ mimicking in the plasma occurring process [14].

Mutual interaction between single RBC has been analyzed based on the so-called depletion interaction model [15]. In this model the interaction between cells is induced by macromolecule depletion near cells due to the balance of entropy and the resulting osmotic pressure pushing cells towards each other, thus forming aggregates [16]. The quantitative calculations of the interaction energies of adhering RBC obtained in the depletion layer model were found to satisfactory match by the AFM and MAT measurements results $[5,7]$.

An alternative model developed for qualitative description of $\mathrm{RBC}$ interaction is based on the concept of the adsorption of macromolecules to membranes of adjacent cells and formation of cross-bridges [17]. This model does not dependent on the relationship between concentration of macromolecules and the cell-to-cell distances. However, inconsistency of the model is in presence of intercellular electrostatic repulsion prohibiting RBC to intimately approach each other for triggering appearance of crossbridges.

Both models are well described in the literature [18-20], and recently, it has been demonstrated that the dextran is associated with depletion-induced cells adhesion, whereas plasma content leads to cross-bridge induced cells adhesion [12,13,21]. These 
interactions are significantly dependent on the size and concentration of dextran and proteins in plasma.

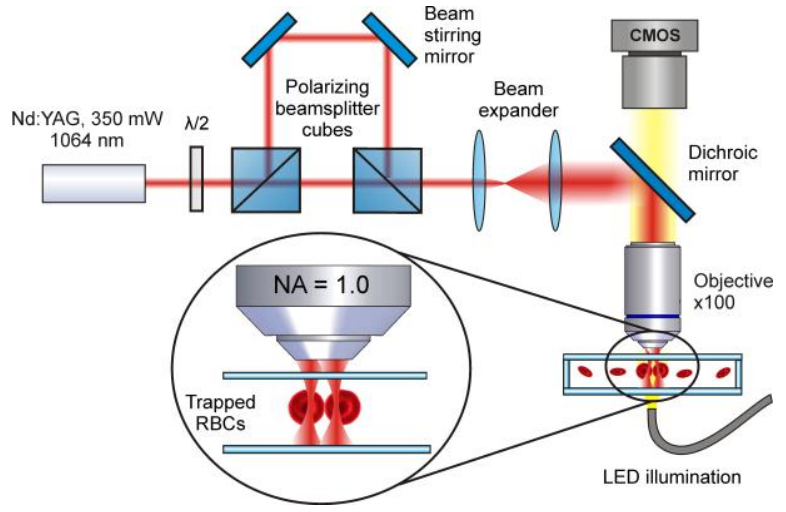

Fig. 1. Schematic presentation of the OT setup for measurements of RBC interaction. Two optical traps are formed with a water-immersion objective $(100 \times, N A=1.00)$. Two interacting $\mathrm{RBC}$ are pulled with two laser beams. The image is formed in transmission mode and registered using a CMOS camera.

The OT-based experimental system developed in-house [12,13] was extensively used for the studies of RBC interaction (Fig.1) both in dextran solutions of different molecular weights, as well as in native plasma solutions. Two optical traps were formed by orthogonally-polarized continuous-wave laser beams from a single-mode Nd:YAG infrared laser $(\lambda=1064 \mathrm{~nm}, 350 \mathrm{~mW}$, ILML3IF-300 Leadlight Technology, Taiwan) and a 100× (NA = 1.00) water-immersion objective (Olympus, LumPlanFI, USA). Laser power in each trap was up to $30 \mathrm{~mW}$. Cells heating was negligible at the given laser power. The traps were positioned by a moving mirror with a translation stage (Thorlabs, USA). Trapped objects were imaged in a transmission mode with a CMOS camera PL-B621M (Pixelink, Canada). Two individual RBC were trapped to measure the interaction energy by OT. The measurements were carried out on 100 pairs of RBC.

To obtain platelet-free blood plasma, the blood was drawn from a clinically healthy donor by venipuncture, transferred into an ethylenediaminetetraacetic acid (EDTA)-covered vial and centrifuged at a speed of $5600 \mathrm{RPM}(3000 \mathrm{~g})$ for $10 \mathrm{~min}$ at room temperature. After that supernatant was collected and centrifuged again under the same conditions. The measurements were performed with the RBC suspended in (1) platelet-free plasma and (2) PBS (Sigma-Aldrich, Germany) dextran solutions: 40, 70, 150 and $500 \mathrm{kDa}$ (Sigma-Aldrich, Germany). Washed RBC were suspended in the platelets-free plasma and in the dextran solutions with the ratio of $0.05 \%$. A chamber used for measurements consisted of two glass plates separated by a $100 \mu \mathrm{m}$ gap made with adhesive tape. About $60 \mu \mathrm{l}$ of the solution were added into the chamber.

The results of the measurements of the RBC adhesion by OT are presented in Fig.2. Following previous studies the measurements of cells interaction were carried out by matching the laser trapping force and with the escape force $[12,13,21]$. In current study, the energy of cells interaction is counted, as measured interaction force normalized to the relative overlapping $\mathrm{RBC}$ area $\mathrm{S}_{\mathrm{i}} / \mathrm{S}_{0}$. The interaction area $S_{i}$ between the cells is defined based on the direct measurements of displacement between to two laser beams and actual size of RBC - $S_{0}$ (see Fig.2). The data are presented as a function of interaction energy per $\mu \mathrm{m}^{2}$ of relative conjugated surface area. To achieve the same viscosity values the concentrations of the dextran solutions were chosen around 1.3 $\mathrm{mPa} * \mathrm{~s}$, as presented in [22]. As one can seen, the interaction energy of mutual interaction of RBC becomes unchanged during the stretching of the cells by OT and independent on the molecular weight of dextran at the given concentrations. Interaction energy show the same dependence for different dextran solutions, and notably differ from those one observed in plasma (see Fig.2).

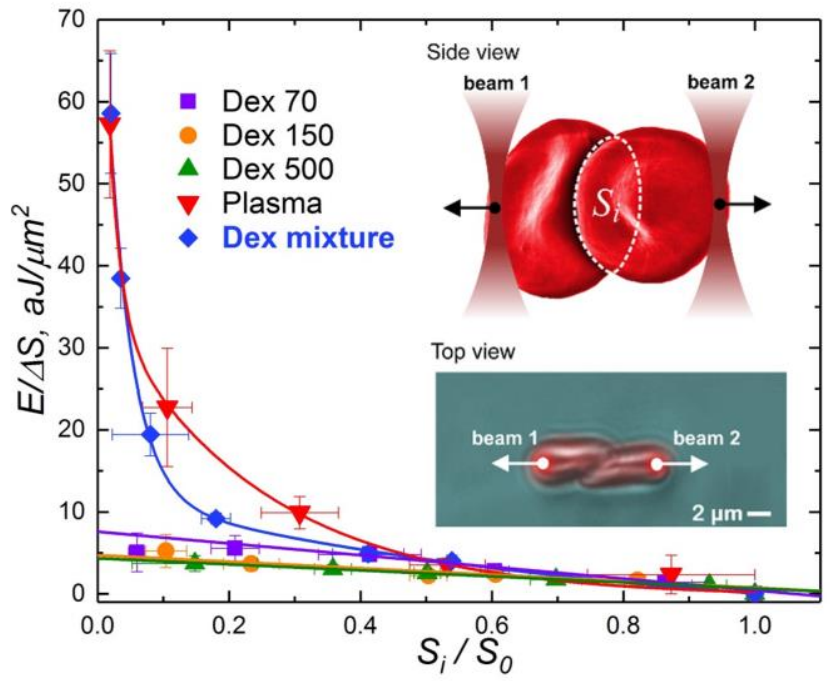

Fig.2. Interaction energy of adhering RBC as a function of relative interaction area $S_{i} / S_{0}$ of conjugated $\mathrm{RBC}$ derived from the measurements in plasma (inverted triangles), dextran mixture (rhombs), and solutions containing dextran molecules of different molecular weights: $70 \mathrm{kDa}$ (squares), $150 \mathrm{kDa}$ (circles), $500 \mathrm{kDa}$ (triangles). Solid lines show the model predictions based on the migrating cross-bridges model for plasma (red), and the depletion interaction model for dextran mixture solution (blue).

In the different dextran solutions with the molecules of same weight the energy of RBC interaction is almost unchanged during the stretching of the cells by OT (see Fig.2). For the dextran solutions at the same viscosity values, the interaction energy was the same. Based on the depletion interaction model [15], this is apparently explained by the fact that osmotic forces are proportional to the interaction area and are uniformly distributed across the cells surface. The depletion layer model combines electrostatic repulsion caused by RBC surface charge and osmotic attractive forces generated by polymer depletion near the RBC surface and penetration of macromolecules into glycocalyx.

Following the depletion interaction model, the interaction energy of adhering RBCs is defined as [15]:

$$
E=-2 p\left(\Delta-\frac{d}{2}+\sigma-z\right)
$$

Here, $p$ is the osmotic pressure, $\Delta$ is thickness of the depletion layer, $d$ is the distance between adjacent surfaces, $\sigma$ is the glycocalyx thickness, and $z$ is the depth of the polymer penetration into the glycocalyx. We found that the mean value of the interaction energy $<E_{\text {dextran }}>=1.2 \pm 0.6 \mu \mathrm{J} / \mathrm{m}^{2}$, observed experimentally by utilizing the OT approach (see Fig.2), agrees well with the value predicted by the depletion interaction model $\left(\sim 1 \mu \mathrm{J} / \mathrm{m}^{2}\right)$ [15].

When RBC are suspended in plasma the interaction energy grows significantly with the stretching of cells by OT and the depletion layer model becomes invalid. In fact, the obtained results 
are in excellent agreement with the predictions based on the migrating cross-bridges model (see Fig.2).

In the migrating cross-bridges model the interaction energy $(E$, $\left[\mu \mathrm{J} / \mathrm{m}^{2}\right]$ ) is defined as [23]:

$$
E=2 k T m_{0} b\left[1+b\left(\frac{s_{i}}{s_{0}}\right)\right]^{-1} .
$$

Here, $S_{i}$ is the conjugated surface area between two interacting cells, $S_{0}$ is the initial interaction (overlapping) area $\left(S_{0}=25 \mu \mathrm{m}^{2}\right), b$ is the dimensionless binding affinity, mo is the initial cross-bridges density before adhesion $\left(1 / \mathrm{nm}^{2}\right), k$ is the Boltzmann constant, $T$ is the absolute temperature $\left(k T=4 \times 10^{-21} \mathrm{~J}\right)$.

Thus, based on the results of OT measurements (see Fig.2) we found values of the binding affinity coefficient $b=11 \pm 5$ and the cross-bridge density $m_{0}=1 / 3600 \mathrm{~nm}^{-2}$.

Blood plasma consists of many types of proteins, which influence aggregation of RBC depending on molecular weights and concentration of main plasma proteins (Table 1).

Table 1. Main plasma proteins composition [24].

\begin{tabular}{|c|c|c|c|c|}
\hline \multicolumn{5}{|c|}{ Plasma proteins } \\
\hline \multicolumn{2}{|l|}{ Protein } & $\begin{array}{l}\text { Concentration, } \\
\text { g/L }\end{array}$ & $\begin{array}{l}\text { M.W., } \\
\text { kDa }\end{array}$ & $\begin{array}{l}\text { Fraction, } \\
\%\end{array}$ \\
\hline \multicolumn{2}{|l|}{ Albumin } & 45 & 66 & 60 \\
\hline \multirow[t]{3}{*}{ Globulins } & $\begin{array}{l}\alpha 1, \\
\alpha 2\end{array}$ & $4.2,6.7$ & 44,85 & \multirow[t]{3}{*}{35} \\
\hline & $\beta$ & 9.1 & & \\
\hline & $\Upsilon \gamma$ & $7-15$ & $\begin{array}{l}(150- \\
850)\end{array}$ & \\
\hline \multicolumn{2}{|c|}{ Fibrinogen } & & 340 & 4 \\
\hline
\end{tabular}

We mixed the dextrans of corresponding molecular weights and volume fraction to the amounts of proteins in plasma: $40 \mathrm{kDa}-$ $8 \mathrm{~g} / \mathrm{L}, 70 \mathrm{kDa}-45 \mathrm{~g} / \mathrm{L}, 150 \mathrm{kDa}$ and $500 \mathrm{kDa}-10 \mathrm{~g} / \mathrm{L}$ with the concentrations proportionally decreased 6 times, i.e.: $40 \mathrm{kDa}-15 \%$, $70 \mathrm{kDa}-60 \%, 150 \mathrm{kDa}$ and $500 \mathrm{kDa}-10 \%$ each. With such mix of concentrations the energy values of RBC interaction taking place an intermediate position between those observed for blood plasma and dextran solutions (see Fig.2). Bearing in mind that the RBC aggregation in dextran solutions and in plasma follows the depletion and cross-bridges interaction model, respectively, we hypothesize that in the case of mixture of dextrans a hybrid model utilizing mechanisms of cross-bridges and depletion should be considered. We emphasize that in a mixture of dextrans with the size ranges and volume fraction proportional to the plasma proteins the dependence of RBC adhering tends close to the cross-bridges model (see Fig.2).

In addition, a SEM imaging was used to visualize the process of the RBC adhesion. RBC incubated either in native plasma or in dextran solutions were fixed with $1 \%$ glutaraldehyde $115 \mathrm{~min}$ at room temperature). A droplet of RBC was dried under vacuum and covered with a $5 \mathrm{~nm}$ layer of platinum. SEM imaging was performed with Zeiss Ultra Plus field emission scanning electron microscope (CarlZeiss, Germany). The results of a direct visualization of the RBC after incubation in blood plasma just before their separation is shown in Fig.3.

It is well known that the cross-bridges are created by protein molecules accumulated between cells. The obtained images clearly show the discrete cilia on the surface of the RBC membrane. As one can see, the cilia are identical and uniformly distributed across the interaction areas (see Fig.3). Density of cilia obtained by SEM imaging (1-2 per $100 \mathrm{~nm}$ distance, see Fig.3) agrees well with the value predicted by the cross-bridges model ( $m_{0} \sim 1.6$ per $\left.100 \mathrm{~nm}\right)$. The actual size of a single cross-bridge is estimated as $\sim 30-40 \mathrm{~nm}$. Thus, the observed cilia can be a product or associated with the protein molecules accumulated between RBC.

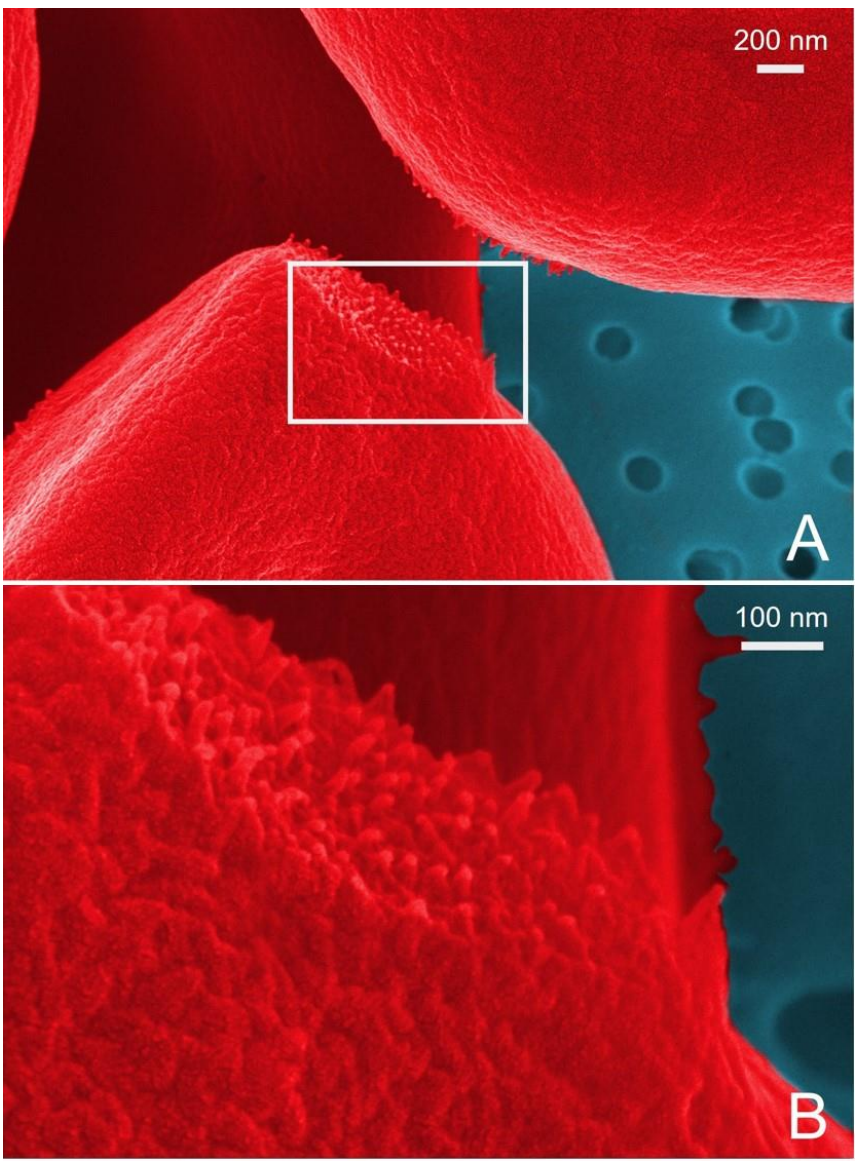

Fig.3. Coloured SEM images of separated RBCs (A). The white rectangle indicates an enlarged area on the cell surface with cilia at the RBC membrane, separately shown in (B).

Figure 4 shows the images of RBC incubated either in $40 \mathrm{kDa}$, in a mixture or in $500 \mathrm{kDa}$ dextran solutions. RBC images obtained from $\mathrm{RBC}$ incubated with $40 \mathrm{kDa}$ dextran (see Fig.4A) and from the dextrans mixture (see Fig.4B) do not demonstrate any differences the polymer solution most likely covers cells in the same way resulting in the connections observed on the images. The $500 \mathrm{kDa}$ dextran molecules are about ten times larger than albumin, the most abundant plasma protein and no discrete cilia are seen anymore and the mechanism of RBC adhesion becomes different (see Fig.4C).

We found that the cross-bridges model is more appropriate for the quantitative description of interaction of the adhering RBC in plasma. This model describes the accumulation of interaction energy due to formation of cross-bridges on adjacent cell membranes. The adhesion of RBC induced both by dextran macromolecules and in native plasma, was observed by direct visualization utilizing field emission SEM.

It has been also found that the density of cilia on the surface of RBC membrane observed by SEM is in excellent agreement with the density of cross-bridges assessed by the OT measurements utilizing 
the cross-bridges model. Thus, we believe that the observed cilia are a product associated with the protein molecules accumulated between RBC that confirms the cross-bridges model. In the dextran solution, which molecules are ten times larger than the most of plasma proteins the cilia are not observed (see Fig.4).
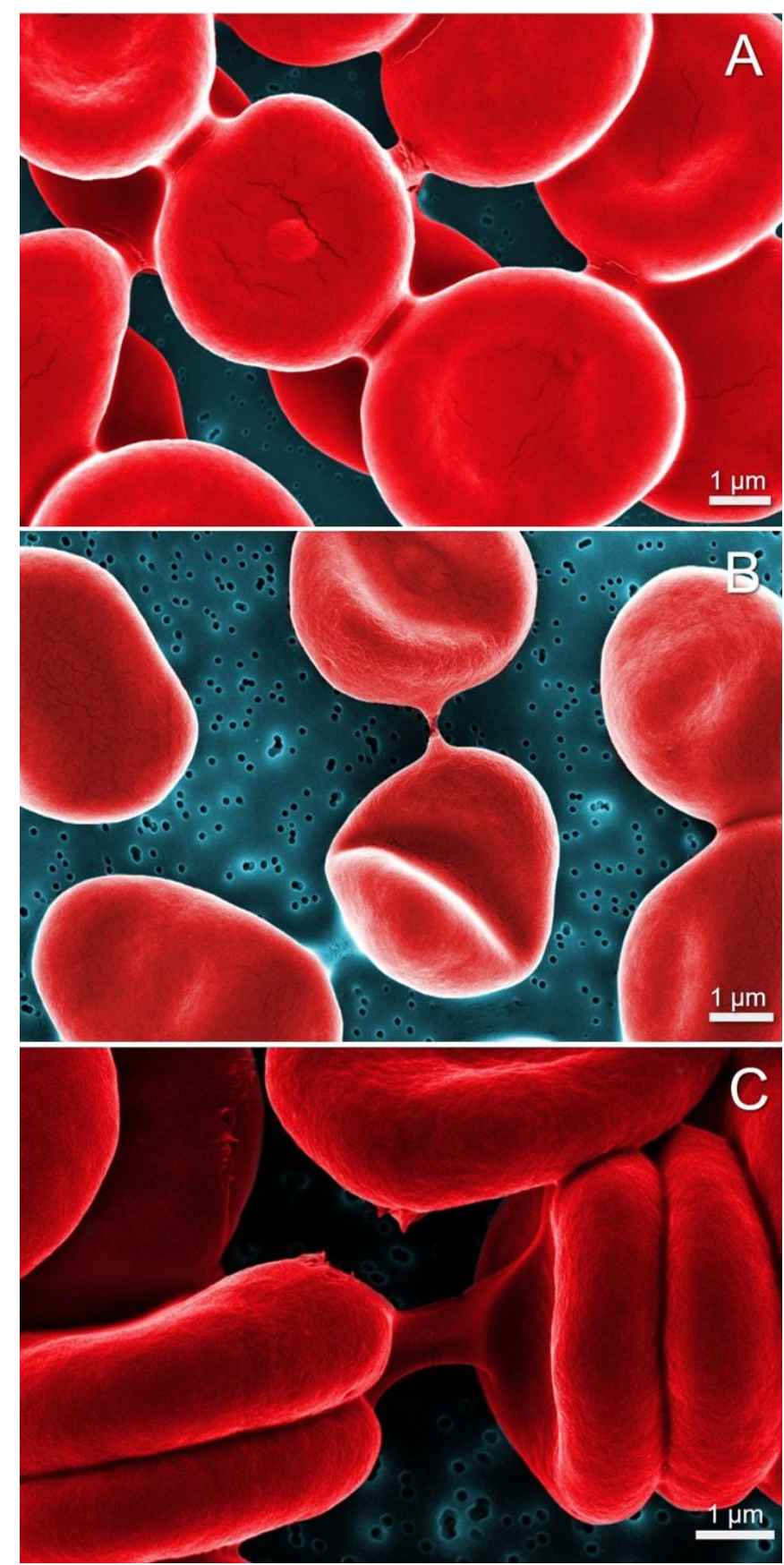

Fig.4. Coloured SEM images RBC in $40 \mathrm{kDa}$ dextran solution (A), in a mixture of dextrans (B) and in $500 \mathrm{kDa}$ dextran solution (C).

In summary, we have demonstrated that OT provide a unique opportunity to obtain new insights into mechanisms of RBC aggregation, and can be used as an effective tool for quantitative assessment of RBC interaction. We have applied the OT technique to investigate interaction of $\mathrm{RBC}$ in dextran solutions and in plasma. The OT approach has been used to measure the interaction energy of the adhering RBC as a function of the relative conjugated surface area. We found that in the dextran solution the interaction of adhering RBC agrees well with the quantitative predictions obtained on the basis of the depletion-induced cells adhesion model. A mixture of dextrans with molecular weights and concentration ratios similar to those of proteins in blood plasma can serve as a plasma model resulting in the similar interaction energy dependence.

Acknowledgment. Authors acknowledge CIMO Fellowship (grant TM-17-10370) and financial support from Academy of Finland (projects 314369, 290596, 311698). Authors also acknowledge initial involvement of Dr. K. Lee and Prof. A.V. Priezzhev from M.V. Lomonosov Moscow State University (Russia) and Dr. A. Karmenyan from Physics Department of National Don Hwa University (Hualien, Taiwan) at the early stage of the OT development.

\section{References}

1. O. Baskurt, B. Neu, H.J. Meiselman, Red Blood Cell Aggregation, CRC Press, Taylor \& Francis Group, Boca Raton-London-New York (2012).

2. A.N. Korolevich, I. Meglinski, Bioelectrochemistry 52, 223 (2000).

3. S. Shin, J.X. Hou and J.S. Suh, Clin. Hemorheol. Microcirc. 42, 117 (2009).

4. O.K. Baskurt, M. Uyuklu, P. Ulker, M. Cengiz, N. Nemeth, T. Alexy, S. Shin, M.R. Hardeman, H.J. Meiselman, Clin. Hemorheol. Microcirc. 43, 283 (2009).

5. K. Buxbaum, E. Evans and D. Brooks, Biochemistry 21, 3235 (1982)

6. S. Chien and K.M. Jan, Microvasc. Res. 5, 155 (1973).

7. P. Steffen, C. Verdier and C. Wagner, Phys. Rev. Lett. 110, 018102 (2013).

8. A. Ashkin, Phys. Rev. Lett. 24, 156 (1970).

9. K.O. Greulich, Rep. Prog. Phys. 80, 026601 (2016).

10. K. Dholakia and T. Cizmar, Nature Photon. 5, 335 (2011).

11. F.M. Fazal and S.M. Block, Nature Photon. 5, 318 (2011).

12. K. Lee, A. Danilina, M. Kinnunen, A. Priezzhev, and I. Meglinski, IEEE J. Sel. Top. Quantum Electron. 22, 7000106 (2016).

13. K. Lee, M. Kinnunen, M.D. Khokhlova, E.V. Lyubin, A.V. Priezzhev, I. Meglinski, and A.A. Fedyanin, J. Biomed. Opt. 21, 035001 (2016).

14. J.K. Armstrong, R. B. Wenby, H.J. Meiselman and T.C. Fisher, Biophys. J. 87, 4259 (2004).

15. S. Asakura and F. Oosawa, J. Polym. Sci. 33, 183 (1958).

16. B. Neu and H. Meiselman, Biophys. J. 83, 2482 (2002).

17. S. Chien, L.A. Sung, S. Simchon, M.M.L. Lee, K.M. Jan, and R. Skalak, Ann. N.Y. Acad. Sci. 416, 190 (1983).

18. P. Steffen, A. Jung, D.B. Nguyen, T. Müller, I. Bernhardt, L. Kaestner, and C. Wagner, Cell Calcium 50, 54-61 (2011).

19. C. Wagner, P. Steffen, and S. Svetina, C. R. Physique 14, 459-469 (2013).

20. B. Neu, R. Wenby, and H.J. Meiselman, Biophys. J. 95(6), 3059-3065 (2014).

21. K. Lee, C. Wagner, and A. Priezzhev, J. Biomed. Opt. 22(9), 091516 (2017).

22. International Committee for Standardisation in Haematology. Recommendation for a selected method for the measurement of plasma viscosity. J Clin Pathol., 37, 1147 (1984).

23. A. Tozeren, K.L.P. Sung and S. Chien, Biophys. J. 55, 479 (1989).

24. F.W. Putnam, The Plasma Proteins: Structure, Function and Genetic Control, 2nd Ed., ed., Academic Press, New York, Vol. 1, p. 141, 147 (1975). 


\section{Full Reference}

1 O. Baskurt, B. Neu, H.J. Meiselman, Red Blood Cell Aggregation, CRC Press, Taylor \& Francis Group, Boca Raton-London-New York (2012).

2 A.N. Korolevich, I. Meglinski, "The experimental study of the potentialities of diffusing wave spectroscopy for the investigating of the structural characteristics of blood under multiple scattering", Bioelectrochemistry 52, 223-227 (2000).

3 S. Shin, J.X. Hou and J.S. Suh, "A transient, microfluidic approach to the investigation of erythrocyte aggregation: the threshold shearstress for erythrocyte disaggregation", Clin. Hemorheol. Microcirc. 42, 117-125 (2009).

4 O.K. Baskurt, M. Uyuklu, P. Ulker, M. Cengiz, N. Nemeth, T. Alexy, S. Shin, M.R. Hardeman, H.J. Meiselman, "Comparison of three instruments for measuring red blood cell aggregation", Clin. Hemorheol. Microcirc. 43, 283-298 (2009).

5 K. Buxbaum, E. Evans and D. Brooks, "Quantitation of surface affinities of red blood cells in dextran solutions and plasma", Biochemistry 21, 3235-3239 (1982)

6 S. Chien and K.M. Jan, "Ultrastructural basis of the mechanism of rouleaux formation", Microvasc. Res. 5, 155-166 (1973).

7 P. Steffen, C. Verdier and C. Wagner, "Quantification of depletioninduced adhesion of red blood cells", Phys. Rev. Lett. 110, 018102 (2013).

8 A. Ashkin, "Acceleration and trapping of particles by radiation pressure", Phys. Rev. Lett. 24, 156 (1970).

9 K.O. Greulich, "Manipulation of cells with laser microbeam scissors and optical tweezers: a review", Rep. Prog. Phys. 80, 026601 (2016).

10 K. Dholakia and T. Cizmar, "Shaping the future of manipulation", Nature Photon. 5, 335-342 (2011)

11 F.M. Fazal and S.M. Block, "Optical tweezers study life under tension", Nature Photon. 5, 318-321 (2011).

12 K. Lee, A. Danilina, M. Kinnunen, A. Priezzhev, and I. Meglinski, "Probing the red blood cells aggregating force with optical tweezers", IEEE J. Sel. Top. Quantum Electron. 22, 7000106 (2016).

13 K. Lee, M. Kinnunen, M.D. Khokhlova, E.V. Lyubin, A.V. Priezzhev, I. Meglinski, and A.A. Fedyanin, "Optical tweezers study of red blood cells aggregation and disaggregation in plasma and protein solutions", J. Biomed. Opt. 21, 035001 (2016).

14 J.K. Armstrong, R. B. Wenby, H.J. Meiselman and T.C. Fisher, "The hydrodynamic radii of macromolecules and their effect on red blood cell aggregation", Biophys. J. 87, 4259-4270 (2004).

15 S. Asakura and F. Oosawa, "Interaction between particles suspended in solutions of macromolecules", J. Polym. Sci. 33, 183192 (1958).

16 B. Neu and H. Meiselman, "Depletion-mediated red blood cell aggregation in polymer solutions", Biophys. J. 83, 2482-2490 (2002).

17 S. Chien, L.A. Sung, S. Simchon, M.M.L. Lee, K.M. Jan, and R. Skalak, "Energy balance in red cell interactions", Ann. N.Y. Acad. Sci. 416, 190-206 (1983).

18 P. Steffen, A. Jung, D.B. Nguyen, T. Müller, I. Bernhardt, L. Kaestner, and C. Wagner, "Stimulation of human red blood cells leads to Ca2+-mediated intercellular adhesion", Cell Calcium 50, 54- 61 (2011).

19 C. Wagner, P. Steffen, and S. Svetina, "Aggregation of red blood cells: From rouleaux to clot formation", C. R. Physique 14, 459-469 (2013).

20 B. Neu, R. Wenby, and H.J. Meiselman, "Effects of dextran molecular weight on red blood cell aggregation", Biophys. J. 95(6), 3059-3065 (2008)

21 K. Lee, C. Wagner, and A. Priezzhev, "Assessment of the "crossbridge"-induced interaction of red blood cells by optical trapping combined with microfluidics", J. Biomed. Opt. 22(9), 091516 (2017).

22 International Committee for Standardisation in Haematology. Recommendation for a selected method for the measurement of plasma viscosity. J Clin Pathol., 37, 1147 (1984).

23 A. Tozeren, K.L.P. Sung and S. Chien, "Theoretical and experimental studies on cross-bridge migration during cell disaggregation", Biophys. J. 55, 479-487 (1989).

24 The Plasma Proteins: Structure, Function and Genetic Control, 2nd Ed., Frank W. Putnam, ed., Academic Press, New York, Vol. 1, p. 141, 147 (1975). 